\title{
Erratum: Heisenberg and Dzyaloshinskii-Moriya interactions controlled by molecular packing in trinuclear organometallic clusters [Phys. Rev. B 95, 094432 (2017)]
}

\author{
B. J. Powell, J. Merino, A. L. Khosla, and A. C. Jacko \\ (Received 1 August 2017; published 20 September 2017)
}

DOI: 10.1103/PhysRevB.96.099902

The forms of the intermolecular spin-orbit coupling discussed in Secs. IV A and IV B of our recent paper do not have the $C_{3}$ symmetry of the underlying molecular system. In particular, $\lambda_{\mu v ; i j}$ is a pseudovector; our original treatment did not properly account for this. Below, we give the correct $C_{3}$-symmetric forms of these interactions and describe the changes in our results when this is corrected.

In Sec. IV A, we studied the case where the intramolecular spin-orbit coupling only couples equivalent Wannier orbitals $(i=j)$ on neighboring molecules $(\mu$ and $\nu)$ by setting $\lambda_{\mu \nu ; i j}=\lambda_{\mu \nu} \delta_{i j}$. The correct $C_{3}$-symmetric form is

$$
\lambda_{\mu v ; i j}=\left(\lambda_{1} \cos \phi_{j}-\lambda_{2} \sin \phi_{j}, \lambda_{2} \cos \phi_{j}+\lambda_{1} \sin \phi_{j}, \lambda_{3}\right) \delta_{i j},
$$

where $\phi_{j}=2 \pi(j-1) / 3$ and $\lambda_{1}, \lambda_{2}$, and $\lambda_{3}$ are constants. Importantly, this form of the intermolecular spin-orbit coupling correctly accounts for the mixing of the $x$ and $y$ components of $\lambda_{\mu v ; i j}$ under the threefold rotations about the $z$ axis. Thus, $\sum_{i, j=1}^{3} \lambda_{\mu \nu ; i j}^{x}=\sum_{i, j=1}^{3} \lambda_{\mu v ; i j}^{y}=0$. It then follows immediately from Eqs. (27) that $D_{\mu \nu}^{x}=D_{\mu \nu}^{y}=0$. Thus, Eq. (28) should be replaced by

$$
\boldsymbol{D}_{\mu \nu}=\hat{z} \frac{8 t_{\mu \nu} \lambda_{3}}{9\left(J_{c}-2 t_{c}\right)},
$$

where $\hat{z}=(0,0,1)$.

In Sec. IV.B, we allowed orbitals to couple to every orbital on neighboring molecules. The most general $C_{3}$-symmetric form of the coupling constant is

$$
\lambda_{\mu v ; i j}=\left[\lambda_{1}(i-j) \cos \phi_{j}-\lambda_{2}(i-j) \sin \phi_{j}, \lambda_{2}(i-j) \cos \phi_{j}+\lambda_{1}(i-j) \sin \phi_{j}, \lambda_{3}(i-j)\right],
$$

where now the functions $\lambda_{1}(i-j), \lambda_{2}(i-j)$, and $\lambda_{3}(i-j)$ only depend on the difference of $i$ and $j$ modulo 3 . Again, one has $D_{\perp}^{x}=D_{\perp}^{y}=0$; indeed this could have been anticipated from the $C_{3}$ symmetry of the problem [1]. Thus, Eq. (29) should be replaced by

$$
\boldsymbol{D}_{\perp}=\hat{z} \frac{8}{9\left(J_{c}-2 t_{c}\right)} \sum_{i j} t(i) \lambda_{3}(j) .
$$

These errors have important consequences for $\mathrm{Mo}_{3} \mathrm{~S}_{7}(\mathrm{dmit})_{3}$ : One finds that $\boldsymbol{D}_{\perp} / \mathcal{J}_{\perp}=0.019 \hat{z}$; similarly, Fig. 9 should be replaced by Fig. 1 below (the curves for $D_{\perp}^{z}$ and $\mathcal{J}_{\perp}$ are unchanged but $D_{\perp}^{x}=D_{\perp}^{y}=0$ ).

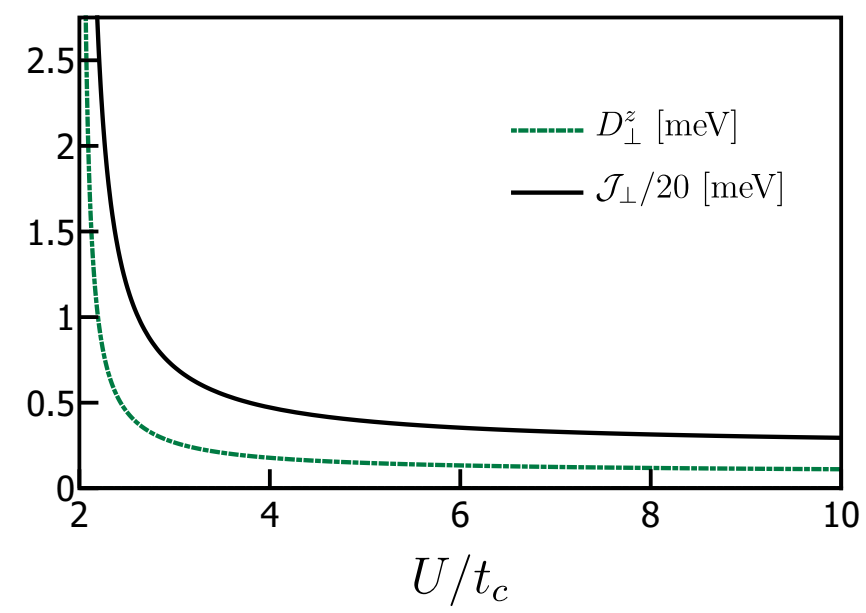

FIG. 1. Effective interlayer Dzyaloshinskii-Moriya and exchange interactions in $\mathrm{Mo}_{3} \mathrm{~S}_{7}(\mathrm{dmit})_{3}$ as a function of the interaction strength. Here we have set $J_{c}=4 t_{c}^{2} / U$ and taken all other parameters from first-principles calculations [2,3]. Note that the perturbation theory breaks down as $U \rightarrow 2 t_{c}$. 
Finally, we note that the above discussion implies that the quantization axis (z) in Eq. (32) is parallel to the crystallographic $c$ axis of $\mathrm{Mo}_{3} \mathrm{~S}_{7}(\mathrm{dmit})_{3}$.

Except as discussed above, the results and conclusions of the paper are unchanged.

[1] T. Moriya, Phys. Rev. 120, 91 (1960).

[2] A. C. Jacko, C. Janani, K. Koepernik, and B. J. Powell, Phys. Rev. B 91, 125140 (2015).
[3] A. C. Jacko, A. L. Khosla, J. Merino, and B. J. Powell, Phys. Rev. B 95, 155120 (2017). 